in the holomorph of $A$. In general, this substitution transforms the holomorph of $G$ into itself; and when $G$ is nonabelian this substitution and the holomorph of $G$ generate the double holomorph of $G$. In regard to the given simple isomorphism between $G$ and $G^{\prime}$ it may be added that if we let every operator of any group correspond to its inverse we get a simple isomorphism only when the group is abelian; but if we multiply in one of these two corresponding groups on the right and in the other on the left there always results a certain kind of simple isomorphism. Moreover, it is easy to verify that if this kind of simple isomorphism can be established between two groups these groups must be actually simply isomorphic.

As regards the theorems relating to conjugate subgroups and invariant subgroups which were developed in $\S 2$, it should perhaps be emphasized that these theorems enable us to determine at a glance all the conjugates of a given subgroup, or of a given operator, provided the potential and the antipotential representation of the group are before us. Similarly they enable us to see at a glance what substitutions and what subgroups are invariant. On the other hand, it should be obsserved that the representation of a given group in the potential and the antipotential form is laborious, so that these theorems appear to be of more theoretical than practical interest.

\title{
THE EQUATION OF PICARD-FUCHS FOR AN ALGEBRAIC SURFACE WITH ARBITRARY SINGULARITIES.
}

BY DR. S. LEFSCHETZ.

(Read before the Southwestern Section of the American Mathematical Society, November 28, 1914.)

1. LET $F_{m}(x, y, z)=0$ be the equation of an algebraic surface of order $m$ with arbitrary singularities, the axes having an arbitrary position, and let

$$
\int \frac{P(x, y, z) d x}{F_{z}^{\prime}}
$$

be an abelian integral of the second kind attached to the 
curve $C_{y}$ in (xz), having for equation $F_{n}=0$. If $p$ is the genus of $C_{y}$, then the periods of this integral, considered as functions of $y$, satisfy a linear homogeneous differential equation of order $2 p: E_{2 p}=0$, called the equation of Picard-Fuchs. Introduced by Fuchs, a complete study of it was made by E. Picard* for the case where the surface has only ordinary singularities, that is, a double curve with a finite number of points triple for both the curve and the surface. As it is as yet unproved that any variety of more than two dimensions can be birationally transformed into one having only "ordinary singularities," it seems important, for the complete generality of investigations bearing upon integrals belonging to varieties, first to extend the most important results obtained by Picard to surfaces with arbitrary singularities, and this is the object of the present note.

2. Let $\omega_{1}, \cdots, \omega_{2 p}$ be a fundamental set of periods for the integral in question, $\omega$ any other period. When $y$ describes a closed path, $\omega$ may be changed into $\omega^{\prime}$, and we have

$$
\frac{d^{k} \omega^{\prime}}{d y^{k}}=\sum_{i=1}^{2 p} m_{i} \frac{d^{k} \omega_{i}}{d y^{k}}
$$

the integers $m$ being independent of $k$; hence the differentiaI equation

$$
\begin{aligned}
& E_{2 p}(y)=\left\|\frac{d^{k} \omega}{d y^{k}}, \frac{d^{k} \omega_{1}}{d y^{k}}, \cdots, \frac{d^{k} \omega_{2 p}}{d y^{k}}\right\|=0 \\
& (k=1,2, \cdots, 2 p)
\end{aligned}
$$

remains unchanged, and if it is written in the form

$$
\sum_{0}^{2 p} p_{i} \frac{d^{2 p-i} \omega}{d y^{2 p-i}}=0 \quad\left(p_{1}=1\right)
$$

the $p$ 's, being uniform everywhere in the $y$ plane and regular at infinity, are rational in $y$.

3. Let $a_{1}, a_{2}, \cdots$ be the winding points of the Riemann surface $C_{y}, Q_{i}$ a cross-cut traced in the $x$-plane from a fixed point $A$ around $a_{i}$ and back to $A$. Any period is of the type

$$
\int \frac{P(x, y, z) d x}{F_{z}^{\prime}}
$$

* Picard-Simart, Traité des Fonctions algébriques de deux Variables, vol. 1. p. 94 . 
the sum being extended to a system of cross-cuts $\Sigma_{ \pm} Q_{i}$ forming a closed path on the Riemann surface. $P$ being taken as a polynomial, this period may cease to be holomorphic where and only where two or more of the winding points coincide. Let $(a, b, c)$ be such a place. When $y$ describes a closed path around $b$, the $a$ 's undergo a substitution, product of several cyclic substitutions having no common letters. Let

$$
\left(a_{1}, \cdots, a_{h}\right)
$$

be one of these cycles. For $y=b$, we have

$$
a_{1}=a_{2}=\cdots=a .
$$

When $y$ describes a closed circle of small radius around $b$ the $a$ 's form the vertices of a regular polygon of $h$ sides having $a$ for center. The axes being arbitrary, the curve $C_{b}$ is not reducible; for otherwise, that is, if all plane sections of $F$ through $(a, b, c)$ were reducible, the surface would be a cone of vertex $(a, b ; c)$ (theorem of Castelnuovo) - a case which we may exclude for the present. To a loop $Q_{1}$ going from $a$ around $a_{1}$ and back to $a$ and joining two sheets 1-2 of the Riemann surface $C_{y}$, there will then correspond a loop $Q^{\prime}$ going from $a$ to a winding point $\alpha$ and joining the same sheets. Furthermore since $a_{1}$ can be brought into coincidence successively with $a_{2}, \cdots, a_{h}$, there are loops $Q_{2}, \cdots, Q_{h}$ joining these two sheets and going from $a$ around $a_{2}, \cdots, a_{h}$. The winding point $\alpha$ may or may not be an ordinary branch point, but in any case, when $y=b, \alpha \neq a$.

4. The integral of $\$ 1$ may have periods of two natures. The first will correspond to cycles formed by the loops $Q_{i} Q_{j}$, the second to cycles formed by the loops $Q_{i} Q^{\prime}$. Let $\omega_{1}, \cdots, \omega_{h}$ be the periods corresponding to the cycles

$$
\left(Q_{1}+Q_{2}, \cdots, Q_{h}+Q_{1}\right)
$$

and $\omega_{1}{ }^{\prime}, \cdots$ the periods corresponding to the cycles

$$
Q_{1}+Q^{\prime}, \ldots
$$

and suppose first that the winding point $\alpha$ comes in coincidence with no other for $y=b$. By the same reasoning as Picard's, ${ }^{*}$ it is easy to show then that when $y$ describes a

* Loc. cit., p. 96. 
closed path around $b, \omega_{i}$ changes into $\omega_{i+1}\left(\omega_{h+1}=\omega_{1}\right)$ and $\omega_{i}^{\prime}$ into $\omega_{i}^{\prime}+\omega_{i}$. Let $\theta^{h}=1$. Then

$$
\left(\sum_{i=1}^{h} \theta^{k i} \omega_{i}\right)(y-b)^{-k / h}=R_{k}
$$

is a uniform function of $y$ in the vicinity of $b$, for all values of the integer $k$. Taking $k=1,2, \cdots, h$ we obtain $h$, uniform functions of $y$, and solving the equations for the $\omega$ 's, we have

$$
\omega_{i}(y)=\sum_{k=1}^{h} \theta^{(i-1) k} S_{k}(y)(y-b)^{k / h},
$$

where the $(S)$ 's are uniform in $y$, near $b$. The period $\omega_{i}{ }^{\prime}$ is of the form*

$$
\begin{aligned}
\omega_{i}{ }^{\prime}= & \sum_{k=1}^{h}(y-b)^{k / h}\left[\varphi_{0 k}{ }^{i}+\varphi_{1 k^{i}} \log (y-b)\right. \\
& \left.+\cdots+\varphi_{a k}{ }^{i}(\log (y-b))^{a}\right] \\
& +\sum_{j}(y-b)^{r_{j}}\left[\psi_{0 j}{ }^{i}+\psi_{1 j}{ }^{i} \log (y-b)+\cdots\right],
\end{aligned}
$$

this being the general form of the integrals of $E_{2 p}=0$ in the neighborhood of the critical point $b$. The $r$ 's are the roots of the fundamental equation relative to $b$ which are not of the form $k / h$. When $b$ describes a closed path this integral is increased by one which contains logarithmic terms, or terms in $(y-b)^{r_{i}}$, as long as all the $\psi^{\prime}$ 's, and all the $\varphi$ 's except $\varphi_{01}{ }^{i}, \cdots, \varphi_{0 h^{i}}{ }^{i} \varphi_{1 h^{i}}{ }^{i}$ do not vanish. As we have seen, $\omega_{i}{ }^{\prime}$ is increased by $\omega_{i}$, and $\omega_{i}$ contains no logarithmic terms nor terms in $(y-b)^{r_{j}}$; hence for $\omega_{i}{ }^{\prime}$ we have the expression

$$
\omega_{i}^{\prime}(y)=\varphi(y)+\sum_{k=1}^{h-1} \frac{S_{k}(y)^{\theta(i-1) k}}{\theta^{k}-1}(y-b)^{k / h}+\frac{S_{h}(y)(y-b)}{2 \pi i} \log (y-b),
$$

$\varphi$ being uniform in $y$ near $b$.

5. We may remark that by replacing $P(x, y, z)$ by

$$
(y-b)^{q} P(x, y, z) \text {, }
$$

$q$ being so chosen that

$$
\frac{P(x, y, z)(y-b)^{q}}{F_{z}^{\prime}}
$$

*E. Picard, Traité d'Analyse, vol. 3, p. 282. The $\varphi$ 's and $\psi$ 's are uniform in the neighborhood of $b$. 
shall be finite in the vicinity of $b$, we obtain an integral having periods $\bar{\omega}=(y-b)^{q} \omega, \omega$ being the period of the integral considered so far, corresponding to $\bar{\omega}$, and that these periods $\bar{\omega}$ are all finite. Hence the functions $\varphi, S$ uniform near $b$, have this point for pole, but not for essential singularity, and therefore: The equation $E_{2 p}=0$ has only regular integrals.

It is seen directly, by considering the circuits on the Riemann surface $C_{y}$, that $\sum_{i=1}^{h-1} \omega_{i}=0$. Hence: In the vicinity of $a$ critical point $b$, there is a certain number of independent periods of the integral

$$
\int \frac{P(x, y, z) d x}{F_{z}^{\prime}},
$$

say $\omega_{1}, \cdots, \omega_{8}$, having this point either for pole or for critical algebraic point, and such that when $y$ describes a closed path around $b$, any period is increased by integral multiples of these. A period non-uniform near $b$ behaves either like $\omega_{i}$ or $\omega_{i}^{\prime}$ or a combination of both. This result really generalizes the one obtained by Picard (loc. cit.). The whole theory of double integrals of the second kind of $F$ can now be developed as in the case of the surface with ordinary singularities. The considerations made there only require that the periods $\omega_{1}, \cdots, \omega_{s}$ be finite near $b$. If this condition is not satisfied for all critical points, we can replace the integral considered by

$$
\int \frac{\varphi(y) P(x, y, z) d x}{F_{z}^{\prime}},
$$

$\varphi$ being a polynomial in $y$ so chosen that the above condition shall be satisfied for all points $b$. If $\rho_{0}, \rho, r$ have the same meaning as with Picard* we have for the number of double integrals of the second kind

$$
\rho_{0}=N-4 p-(m-1)+2 r-(\rho-1),
$$

the same formula, if we set $N=\Sigma s$, as that obtained by the French mathematician. $N$ denotes the class when the surface has only ordinary singularities. Since $\rho_{0}$ is an absolute invariant with respect to birational transformations, we have by taking this number for the surface $\bar{F}$ birationally transformed of $F$ and with ordinary singularities

* Picard-Simart, loc. cit., vol. 2, p. 408. 


$$
N-4 p-(m-2)+2 r-\rho=\bar{I}+2 r-\bar{\rho}+2,
$$

where $\bar{\rho}, \bar{I}, \bar{\rho}$ are the invariants $\rho$ and those of Zeuthen-Segre for $\bar{F}$. If $I$ is the same invariant for $F$, since $\bar{I}-\rho=I-\rho$, we have

$$
I=N-4 p-m=\Sigma s-4 p-m .
$$

Hence $\Sigma s$ is equal to the "equivalence" in nodes of the point $(a, b, c)$ in the evaluation of the invariant $I$ for $F$ by means of the pencil $C_{y}$. This property can be shown directly for the following cases: $1^{\circ} . F$ has only ordinary nodes. $2^{\circ}$. In the vicinity of any of these nodes there lie other nodes, or ordinary infinitesimal multiple curves. In these cases it is easy to show that in the vicinity of the nodes all the numbers such as $h$ are equal to 2 . It would be interesting to know if such is always the case, but the preceding investigation shows that for the applications this does not matter.

UNIVERSITY OF KANSAS, October 17, 1914.

\section{THE FOURTH DIMENSION.}

Geometry of Four Dimensions. By Henry Parker Manning, Ph.D. New York, The Macmillan Company, 1914. 8vo. 348 pp.

EvERY professional mathematician must hold himself at all times in readiness to answer certain standard questions of perennial interest to the layman. "What is, or what are quaternions?" "What are least squares?" but especially, "Well, have you discovered the fourth dimension yet?" This last query is the most difficult of the three, suggesting forcibly the sophists' riddle "Have you ceased beating your mother?" The fact is that there is no common locus standi for questioner and questioned. To the professional mathematician the fourth dimension usually suggests a manifold of objects depending upon four independent parameters, which it is convenient to describe in geometrical language. Occasionally he does not make any use of analysis, but builds up what the Italians call a "Sistema ipotetico deduttivo" of abstract assumptions and conclusions. The whole thing is professional, and unromantic. Such ideas, are, naturally, 